\title{
Development of Face Recognition Based Smart Door Lock
}

\author{
Sarah Abdullah \\ American university of the Middle East \\ Kuwait, Kuwait
}

\begin{abstract}
Nowadays, there is a growing interest in the smart home system using the Internet of Things. One of the important aspects of the smart home system is the security capability which can simply lock and unlock the door or the gate. This paper proposed a face recognition security system using Raspberry Pi which can be connected to the smart home system. The components that we will use for this solution are Raspberry pi, camera, Blynk app, and SD card. The output of the face recognition algorithm is connected to a relay circuit, in which it will lock or unlock the magnetic lock placed at the door. Results showed the effectiveness of our proposed system, in which we obtain around $90 \%$ face recognition accuracy. We also proposed a hierarchical image processing approach to reduce the training or testing time while improving the recognition accuracy.
\end{abstract}

Key Words: Face recognition; Smart security System; Control System; Raspberry Pi.

\section{INTRODUCTION}

Significant developments in the field of digital sciences in recent years have given great impetus to the application of control engineering systems in everyday life. This development has made available systems capable of in-depth data analysis, including images and videos. One of the applications of this has become the face recognition technology [1]. Face recognition is a part of the biometrics area, which also contains fingerprint, eye iris recognition, auricle shape, hand geometry, palm lines, the pattern of keystrokes on a keyboard, signature, and speech, and even DNA comparison. This technology enables the identification and verification of a person's identity by comparing facial features with the data stored in the database. Like a fingerprint, face recognition analyzes the textures and shapes [2]. However, unlike many of the above methods, face recognition does not require contact. The system can detect a human face in the form of an image or a video, and it can compare and analyze the predetermined characteristics of the face stored in the database. Ease of use has made this technology very popular for different purposes, such as device locking system, national security systems. Furthermore, the development of low-cost electronics components has made it possible to use this technology in everyday life for various purposes. This research aims to develop face recognition algorithms to design and construct a smart, cost-effective door locking system.

In the remainder of this article, firstly we review literature and categorize the schemes that implements a Face Recognition Based Smart Door Lock in Sec. 2. Then, in Sec. 3, we show the design of our proposed system that consists of raspberry pi 3, camera, solenoid, LEDs, and buzzer. In the same section we show the steps of proposed algorithm. Results of the implemented prototype is shown in Sec. 4. The conclusion and a plane for future work is summarized in Sec.5.

\section{LITERATURE REVIEW}

Face recognition techniques could be divided into two main categories: face recognition from still image and recognition from a video. In this section, face recognition based on still-image will be considered. Zhao et al. have divided the face recognition process into three main parts: pre-processing 'face detection', feature extraction, and, finally, classification or recognition as shown in Figure 1. Each of the steps needs a set of approaches to be performed to complete the task. The authors highlighted that each of the steps must be performed automatically to keep the system effective. As it was said before, each step has its subtasks, for instance, for detection must be performed with consequent normalization of the image. In contrast, the feature extraction step may need a restoration subtask if the image is inaccurate or damaged [1]. Different methods can be used to detect and validate the human face, and an appropriate model can be selected by reviewing the models such as feature-based, knowledge-based, and appearance-based and template matching. 


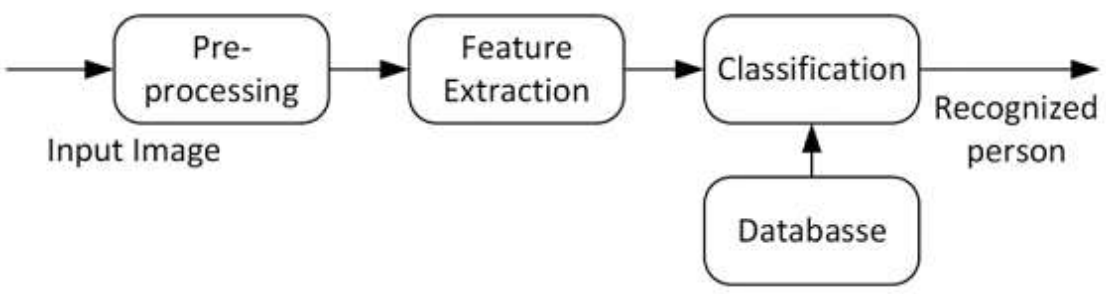

Figure 2. Face recognition subsections.

Hsu et al. have conducted an excellent overview of techniques used to detect faces. According to the authors, face detection is a crucial step in identity identification, due to the importance of the operation and the complexity of many of the techniques, which require large computer calculations [3]. This is due to the high dynamism and high level of facial variability. These are the major challenge for face detection. Therefore, several papers [4-7] were dedicated to the analysis of different approaches to face detection.

The recent years the facial recognition based on video has gained more interest in comparison to still facial images. The videobased recognition aims to find out and extract face features automatically by using face recognition and identification from a video set. Zhao et al. [1] provided fundamental knowledge about video-based face recognition. Then they have categorized already existing approaches of video-based face recognition by given descriptions of methods in detail. Also, they reviewed three main techniques of video-based face recognition by considering the lasted developments that have been done in this area. The given three methods:

- Face segmentation and pose estimation

- Face tracking

- $\quad$ Face modeling

Even though video-based face recognition has more advantages over still-image recognition, there are significant challenges, such as the low quality of the video, small face images, and the characteristic of human body parts [1]. There are research works concentrated on low-level characteristics and the received coefficients from decomposition [8], which is being dealt with low quality of the video. These mentioned methods are instructed on sets of data by labeling manually. However, using these methods is not constructed for recognition tasks related to low-level characteristics. One of the ways to prevent the low quality of the video is to consider an up-to-date method proposed by Wang [9]. In the given paper, the author not only proposed a hybrid approach by learning the function of quality related to an image; he also conducted experiments by demonstrating image quality compared to other methods.

Based on past studies, it can be concluded that the image quality is a critical parameter and significantly affects success. This parameter is especially crucial for the system as a specialized camera was not used, instead of a camera of the phone. However, it is worth noting that the flagship phones have nowadays very high-quality cameras that give a clear image.

\section{SYSTEM DESIGN OF FACE RECOGNITION SYSTEM USING RASPBERRY PI}

In this section, we will show a comparison between three available solutions for a smart door lock and the reasons that leads to the decision for implementing the face recognition one. Also, we give a detailed description about the hardware design and software design used in this work.

\subsection{Available Solutions for a Smart Door Lock}

The idea of our project is to design a smart door system that can apply to homes. So, we need to create a system that can detect the person who knocks on the door. Then it will recognize the face and send it to the owner of the house by an application. After that, the owner will accept or reject that person by a lock or unlock the door. We have three solutions to implement our idea.

The first solution is to make the house door lock with the face recognition process. The face recognition method is a method that will help to identify different facial features by their photos and compare them with saved data on the system. Also, it's a safety system because nobody can access it without taking his picture. Furthermore, it is a reliable system that no one can put any image 
instead of his face. Also, the image processing of this method is accurate, and nobody can hack the system. On the other hand, face recognition has disadvantages, such as the change in the facial features, which leads to weakness process of face recognition. Also, the similarities between the facial features will keep the system unsafe. However, wearing sunglasses will obstruct the process of face recognition and make it unclear. So, the components that we will probably use for this solution are Raspberry pi, power supply, USB camera, Blynk app, SD card, and Arduino Uno. The benefit of using the Blynk app is to receive the notifications of the system immediately. And it will help the owner to observe what happened directly.

Second solution that we found is lock and unlock the door using fingerprint scanner. There is a scanner that takes the fingerprint of the homeowners and saved it in the database, so when the unknown person come and scan their fingerprint, the device will match it with the fingerprints which are stored in the data base memory of the device, if the fingerprint did not match the stored fingerprint a message will be sent to the homeowner through his phone. For this solution we will need fingerprint scanner, Arduino UNO, power supply, Android app Blynk, SD card and Raspberry Pi. One of the advantages of this solution is that it is easy and uncomplicated for the elderly, it considered safe and secure since no one can take your fingerprint, but in the other hand the disadvantage is that some time the scanner will not recognize the fingerprint either if your fingerprint stored in the database, there is many reasons for that like dirty fingerprint scanner or oily fingers. The fingerprint lock system is expensive so it will not be affordable for all classes of society.

The third solution can be found via the home automation using mobile device project. It is one of the papers I read in the literature review. I'm not necessarily using the whole paper to embed in our open sesame like project. It is more like using it as our inspiration for the application link within it. The general idea of this invention paper is the well-established connection between certain home appliances and the mobile device or to be specific, the intended application. What it does is that it sends a processed request from the mobile device to the intended home appliance device, but in our case, we want it for our door and security system in general. After all, the door is symbolized as the beginning and the ending. That indicates our undertaking is open for future evolving, just like a well-trained Pokémon. The needed components are device controller and a wireless transceiver, but the component's slot is not fully closed since these components allowing only proximity due to near field wireless communication protocol. That is one big disadvantage to our own project. The need to be within the household range defeats one of our objectives we expect from the glorified idea, and the soon to be become a reality, in our mindsets. On the other hand, there is the advance computing functionality for the simplest tasks within the app intended for the door and security system. Its counterpart in the disadvantage is the system of home appliances related to security are hard to upgrade. They are about a decade behind the technology of apps and mobile devices. We are hopeful and we are determined to reach the perfect solution within the set time limit of one year, while juggling with what we think the closest solutions to the objectives we set. After a great analyze of the different tradeoffs/compromises of the three available solutions a cording to a predefined requirements and criteria, like safety, reliability, cost, connection, and novelty the decision was to use the first solution which depends on the concept of face recognition process.

\subsection{Hardware Design}

Figure 2 shows the proposed block diagram of face recognition system using Raspberry Pi 3. The Raspberry Pi 3 is connected to the camera module. Raspberry Pi has the same processing capability of a single core processor with built-in graphics which can support up to 1080p video standard using its High-Definition Media Input (HDMI) port. The relay circuit is connected to Raspberry Pi and provides an interface to the high voltage door lock solenoid. The status could be shown using two LED indicators and a buzzer as part of smart home system. The green LED indicates that the detected image is well recognized, while the red one indicates the misdetection. Finally, the power required is less than 1 A, so that a compact power supply, i.e., smartphone charger, could be used as the power supply. The hardware connection is depicted in Figure 3.

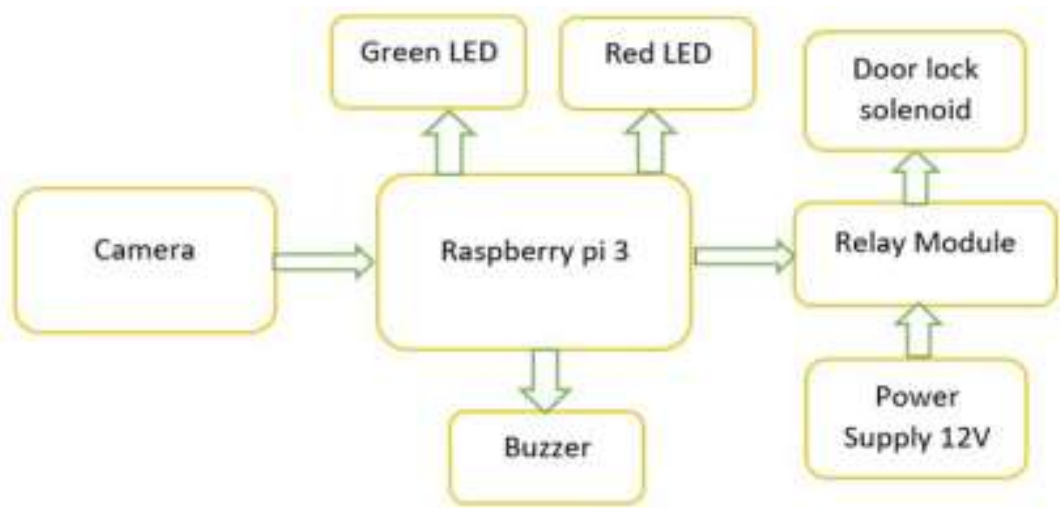

Figure 2. Block Diagram of Proposed Hardware 


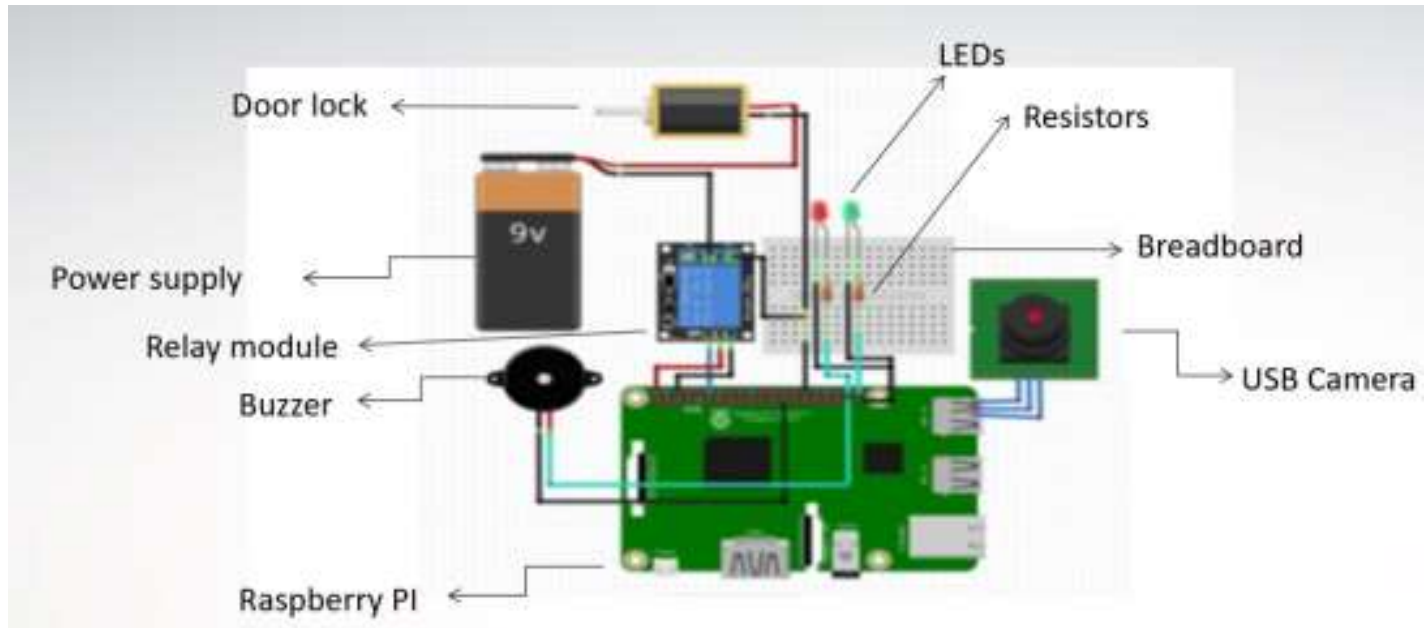

Figure 3. Hardware connection.

\subsection{Software Design}

Figure 4 shows the flowchart of the proposed face recognition system. If the human presence is detected, then the camera will capture the face image. The face detection routine will localize and segment the face region only. The face image is then fed into the face recognition routine. If the recognized face is detected, the system will unlock the door by turning of the magnetic lock. After 30 seconds (configurable), the system will lock again the door by turning on the magnetic lock. The system will then start from the beginning.

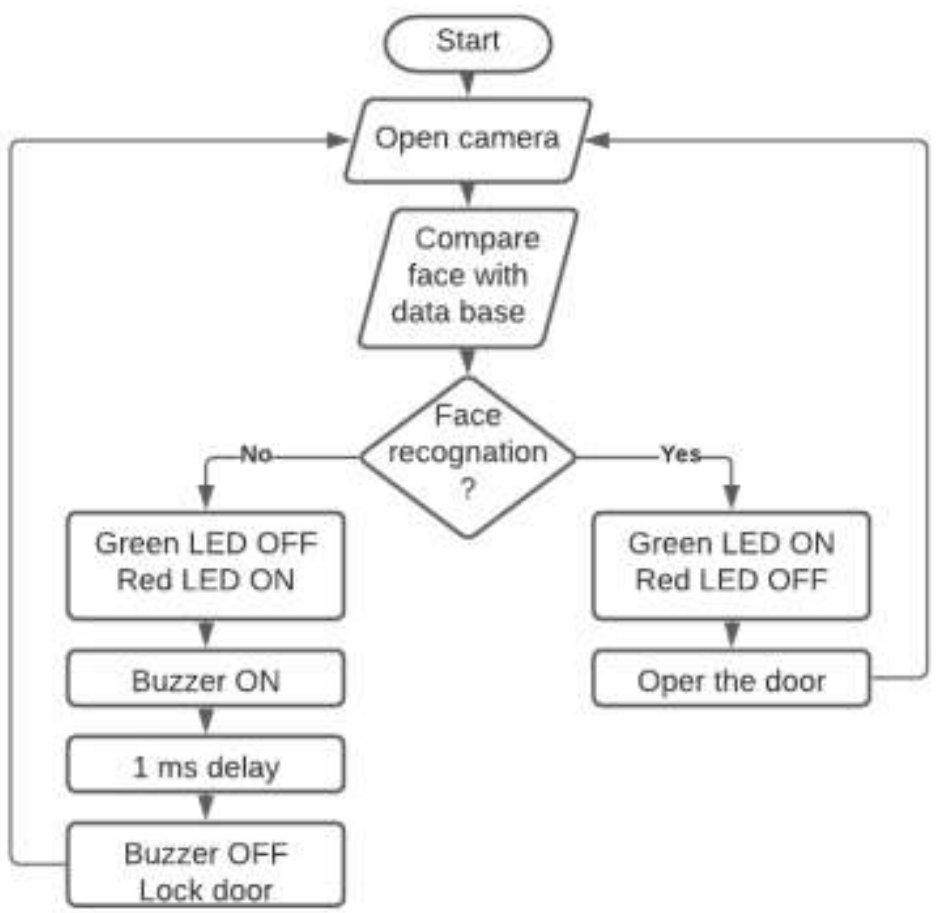

Figure 4. Flowchart of Proposed software

\section{RESULTS}

The model of an intelligent door locking system was constructed. To train a recognition model, at first dataset must be created algorithm and then applied on an extracted face picture. This system was tested on four teammates and provided good enough recognition accuracy. The system demonstrates the result of recognition on the green and red LEDs. The system is very dependent on light quality and the presence of a single-color background. A sample of the carried-out experiments is shown in Figure 5. 


\section{CONCLUSIONS}

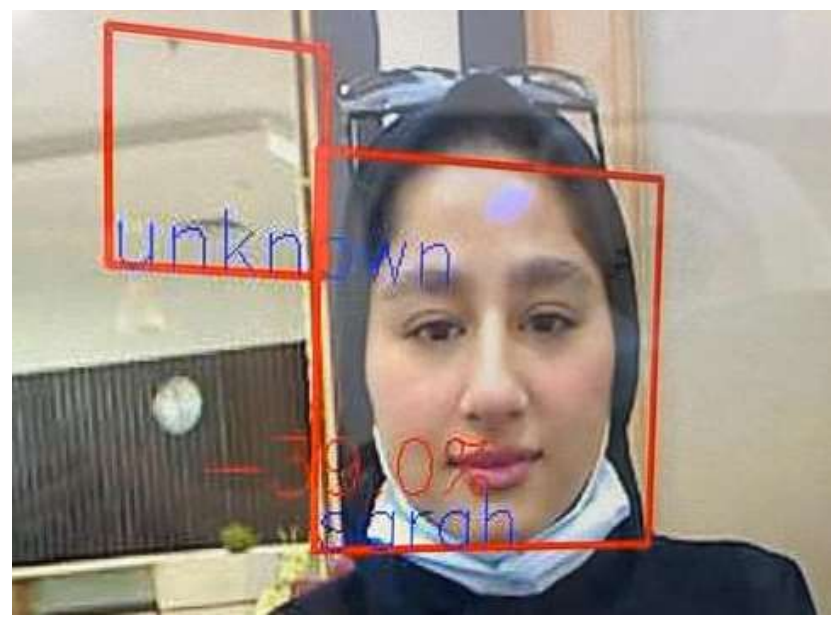

Figure 5. System Test: Face Detection and Recognition.

This paper has presented a face recognition security system using Raspberry Pi 3. The prototype design for real world implementation has been elaborated, in which the output of face recognition algorithm will lock or unlock the magnetic lock placed at the door using relay circuit. This proposed system could be connected using Internet to the smart home system for the added security capability. Further research includes optimization of hierarchical image processing, use different features extraction and classifier, or use parallel Raspberry Pi clusters to speed up the computation.

\section{REFERENCES}

[1] W. Zhao, R. Chellappa, P. J. Phillips, and A. Rosenfeld. 2003. Face recognition: A literature survey. ACM Comput. Surv. 35, 4 (December 2003), 399-458. DOI:https://doi.org/10.1145/954339.954342.

[2] Li, S. Z., Jain, A. K., \& Recognition, F. (2011). Handbook of Face Recognition. Handbook of Face Recognition, $1-15$. https://doi.org/10.1007/978-0-85729-932-1

[3] Hsu, R. L., Abdel-Mottaleb, M., \& Jain, A. K. (2002). Face detection in color images. IEEE transactions on pattern analysis and machine intelligence, 24(5), 696-706.

[4] Pentland, A., Moghaddam, B., \& Starner, T. (1994). View-based and modular eigenspaces for face recognition.

[5] Bergasa, L. M., Mazo, M., Gardel, A., Sotelo, M. A., \& Boquete, L. (2000). Unsupervised and adaptive Gaussian skin-color model. Image and Vision Computing, 18(12), 987-1003.

[6] Lew, M. S., \& Huijsmans, N. (1996, August). Information theory and face detection. In Proceedings of 13th International Conference on Pattern Recognition (Vol. 3, pp. 601-605). IEEE.

[7] Smeraldi, F., Carmona, O., \& Bigün, J. (2000). Saccadic search with Gabor features applied to eye detection and real-time head tracking. Image and Vision Computing, 18(4), 323-329.

[8] Huitao Luo, "A training-based no-reference image quality assessment algorithm," in Proc. 2004 Int. Conf. Image Process. (ICIP’04). IEEE, 2004, vol. 5, pp. 2973-2976.

[9] Wang, C. (2017, December). A learning-based human facial image quality evaluation method in video-based face recognition systems. In the 2017 3rd IEEE International Conference on Computer and Communications (ICCC) (pp. 1632-1636). IEEE. 